\title{
Die Rolle des Bergmännischen Verbandes Österreichs
}

\author{
Gerhard Mayer
}

Lehrstuhl für Bergbaukunde, Bergtechnik und Bergwirtschaft, Montanuniversität Leoben, Leoben, Österreich

Online publiziert 24. Februar 2020

\begin{abstract}
Zusammenfassung: In der Zeitspanne von 1997-2020, also seit der Übernahme der Geschäftsführung durch den Autor, ist der Bergmännische Verband Österreichs von einer starken Erweiterung seines Tätigkeitsfeldes und steigenden Mitgliederzahlen gekennzeichnet. Die Struktur der Organisation des BVÖ und seine Aktivitäten auf technischwissenschaftlichem und wirtschaftlichem Gebiet werden erläutert, die Beziehungen zu nationalen Behörden und Einrichtungen dargestellt. Die Entwicklung des Verbandes geht angesichts der aktuellen Probleme der Sicherung der Mineralstoffgewinnung und -versorgung in Richtung verstärkter Zusammenarbeit mit Universitäten und Institutionen im europäischen Raum und darüber hinaus.
\end{abstract}

Schlüsselwörter: BVÖ Bergmännischer Verband Österreichs, Entwicklung 1997-2020, Struktur des BVÖ, Aktivitäten, Nationale und internationale Verbindungen

The Role of the Austrian Mining Association (BVÖ)

Abstract: During the period 1997-2020, i.e. under the author's management responsibility, the Austrian Mining Association (BVÖ) has been characterized by a significant extension of its scope of activities and increasing membership. The Association's organizational structure and activities in technical, scientific and economic areas are described, its relations to national authorities and institutions are reviewed. In response to the current problems of ensuring mineral resource production and supply, the Association's development is heading for enhanced cooperation with universities and institutions in Europe and beyond.

Keywords: Austrian Mining Association (BVÖ), Development 1997-2020, BVÖ structure, BVÖ activities, National and international relationships

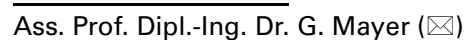

Lehrstuhl für Bergbaukunde, Bergtechnik und Bergwirtschaft, Montanuniversität Leoben,

Franz-Josef-Straße 18,

8700 Leoben, Österreich

gerhard.mayer@unileoben.ac.at

\section{Einleitung}

Dieser Artikel bietet eine Darstellung des Bergmännischen Verbandes Österreichs von 1997 bis zur Gegenwart, also schwerpunktmäßig die Zeitspanne, in der die Geschäftsführung in den Händen des Autors gelegen ist. Die Thematik der nachhaltigen Versorgung mit mineralischen Rohstoffen rückt immer mehr in den Fokus von Gesellschaft, Wissenschaft und Wirtschaft. Der BVÖ fördert in Zusammenarbeit mit internationalen Universitäten und Partnerorganisationen die Entwicklung und den Informationsaustausch auf technisch-wissenschaftlichem Gebiet und umfasst dabei ein breites Spektrum von Lagerstättenforschung, Gewinnung untertage und im Tagbau, Aufbereitung, Markscheidewesen und Bergschäden. Die Fachausschüsse des BVÖ haben darüber hinaus auch die Aufgabe, fachliche Ausbildung und Weiterbildung der Verbandsmitglieder, im Besonderen auch für Studierende und Schüler, zu organisieren und bereitzustellen.

Im Folgenden ein Überblick über die gegenwärtige Struktur und die Aktivitäten des BVÖ und ein Ausblick in zukünftige Entwicklungen.

\section{Geschichtlicher Abriss}

Am 7. Oktober 1950 wurde der "Verband der Bergingenieure Leoben“ gegründet, dem Vertreter der Behörde, der Montanistischen Hochschule und der Industrie angehörten.

Zwei Jahre später wurde der Verband, der in erster Linie die Aufgabe hatte, eine Berufs- und Standesorganisation der Bergingenieure Österreichs zu sein und diese in der Öffentlichkeit und auch gegenüber den Arbeitgebern zu vertreten [1], in „Verband der Bergingenieure Österreichs" umbenannt.

Die Hauptversammlung 1963 beschloss eine neue Satzung, welche als Vereinszweck die gemeinnützige Förderung des österreichischen Bergwesens und der damit verbundenen Wirtschaftszweige auf wissenschaftlichem, technischem und wirtschaftlichem Gebiet definierte und ihm den Namen „Bergmännischer Verband Österreichs" gab (Abb. 1).

Durch die neue Satzung wurde der Kreis der Verbandsangehörigen insofern erweitert, als nun auch Unternehmen 
Abb. 1: Bescheid vom 11. Juli 1963

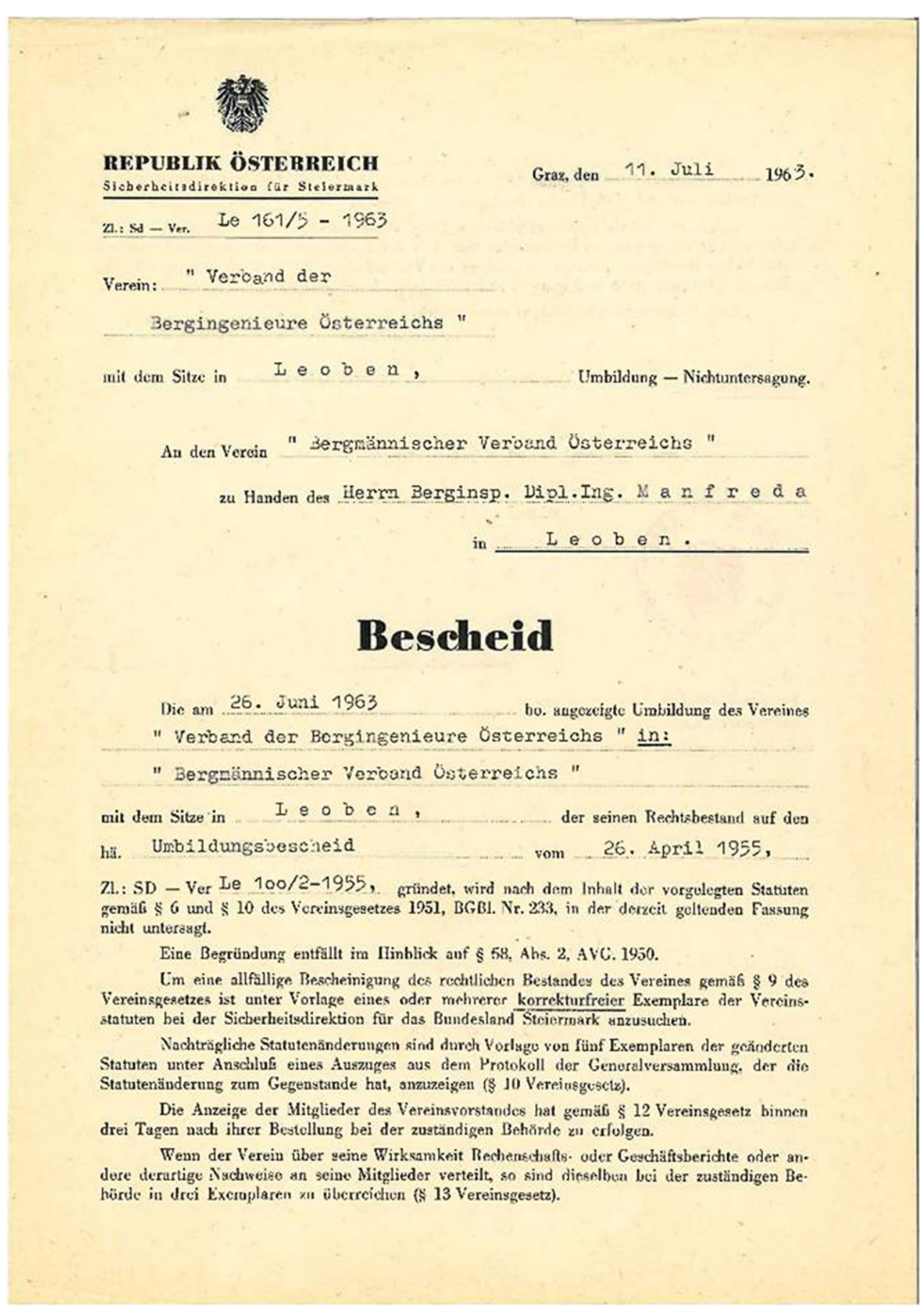

REPUBLIK ÖSTERREICH

11. Juli den 1963.

D.: su - Ver. Le $161 / 5-1963$

"Veroand der

rgingenieure Osterreichs

dem Sitze in I e o b e $n$

Umbildung - Nichtuntersagung.

zu Handen des Herm Berginsp. Dipl.Ing. M a $n f r e d a$ in $\quad \mathrm{L} \in \mathrm{O} \mathrm{b} \in \mathrm{n}$. der Rohstoffindustrie Mitglieder werden konnten, wodurch sich innerhalb kurzer Zeit die Zahl der Mitglieder auf 800 verdoppelte, darunter alle maßgeblichen Unternehmen der Mineralrohstoffgewinnung in Österreich [2].

Im Jahr 2020 feiert der Bergmännische Verband Österreichs somit sein 70jähriges Bestehen.

\section{Struktur des Verbandes}

- Das Präsidium des BVÖ besteht aus dem Präsidenten und bis zu 3 Vizepräsidenten. Bei der Wahl der Präsidenten gilt seit jeher das Prinzip, dass Angehörige der Universitäten, der Fachbehörden und der Industrie einander abwechseln. 
- Als Funktionäre agieren

der Geschäftsführer mit Sekretärin,

der Schatzmeister und der Schriftführer.

- Dem Vorstand obliegt die verantwortliche Leitung des Verbandes, er vertritt diesen in gerichtlichen und außergerichtlichen Angelegenheiten.

- Als Operatives Gremium fungiert der Vorstandsausschuss.

- Ziel der Fachausschüsse ist die Pflege des Erfahrungsaustausches auf technisch-wissenschaftlichem Gebiet, die Entwicklung sowie die schnelle Verarbeitung neuer Erkenntnisse, Informationsaustausch und fachliche Fortbildung. Fachausschüsse dienen damit gleichzeitig der Ausbildung und Weiterbildung der Verbandsmitglieder. Derzeit sind folgende Fachausschüsse des BVÖ eingerichtet:

Fachausschuss für Lagerstättenforschung,

Fachausschuss für untertägigen Bergbau,

Fachausschuss für Aufbereitung,

Fachausschuss für Markscheidewesen (einschl. Bergschäden),

Fachausschuss für Tagbau- und Steinbruchtechnik,

Fachausschuss für Studenten \& Schüler.

- Mitglieder können sein:

Einzelpersonen, Behörden, Institute, Firmen und sonstige Körperschaften (Ordentliche Mitglieder),

Pensionisten,

Studenten (Außerordentliche Mitglieder),

Ehrenmitglieder. Mit der Verleihung der Albert Millervon-Hauenfels-Medaille, die im Jahr 1957 vom BVÖ für Verdienste um den Bergbau gestiftet wurde [2], ist eine Ehrenmitgliedschaft verbunden.

\subsection{Präsidenten des BVÖ}

Seit seiner Gründung standen dem BVÖ insgesamt 25 Präsidenten vor, seit 1997 bis dato sind es 8:

- Bergrat h.c. Dipl.-Ing. Dr. mont. Klaus MOLDAN (Industrie)

- O. Univ.-Prof. Dipl.-Ing. Dr. mont. Horst WAGNER (Montanuniversität)

- Geschäftsführer, Bergrat h.c. Dipl.-Ing. Dipl.-Ing. Dr. mont. Josef KORAK (Industrie)

- Vice-President Bergrat h.c. Dipl.-Ing. Dr. mont. Manfred HOSCHER (Industrie)

- Sektionschef a.D. Mag. iur. Dipl.-Ing. Dr. mont. Alfred MAIER (Behörde)

- Ingenieurkonsulent für Bergwesen Dipl.-Ing. Martin Erich LANG (Industrie)

- Bereichsleiter Ministerialrat Dipl.-Ing. Mag. iur. Arthur MAURER (Behörde)

- seit 28.05.2019: Bergrat h.c. Dipl.-Ing. Josef PAPPENREITER (Industrie)

\begin{tabular}{|llll|}
\hline \multicolumn{2}{|l}{ TABELLE 1 } \\
Entwicklung der Mitgliederzahlen des BVÖ & 1997-2020 \\
\hline Jahr & Personen & Firmen & Gesamt \\
\hline 1997 & 742 & 86 & 828 \\
\hline 2020 (Februar) & 1005 & 71 & 1076 \\
\hline
\end{tabular}

\subsection{Entwicklung der Mitgliederzahlen des BVÖ}

Die Gesamtzahl der Mitglieder hat seit 1997 eine sehr konstante Entwicklung mit einer Steigerung von etwa $25 \%$ ab 2011 erfahren.

Seit der Gründung des Fachausschusses für Studenten und Schüler im April 2015 hat sich allerdings deren Mitgliederzahl von damals ca. 125 auf derzeit 260 Studierende mehr als verdoppelt, die Tendenz ist steigend (Tab. 1).

\section{Aktivitäten des Verbandes}

Der Vereinszweck der Förderung des österreichischen Bergwesens und der damit verbundenen Wirtschaftszweige auf wissenschaftlichem, technischem und wirtschaftlichem Gebiet auf gemeinnützige Weise wird gemäß den geltenden Statuten mittels folgender Aktivitäten erfüllt:

- Persönliche Fühlungnahme der Verbandsmitglieder zum Austausch von Erfahrungen und Erkenntnissen,

- Veranstaltung von Vorträgen, Fachtagungen und Fachkursen sowie von Exkursionen und Studienreisen,

- Tätigkeit von Fachgruppen, Fachausschüssen und Arbeitskreisen zur Bearbeitung wissenschaftlicher, technischer und betrieblicher Fragen,

- Herausgabe eines eigenen Schrifttums und die Veranlassung und Förderung von Veröffentlichungen der Verbandsmitglieder,

- Förderung von Lehre und Forschung.

\subsection{Tagungen}

Seit 1997 hat der Bergmännische Verband 63 Tagungen ausgerichtet, nämlich etwa drei pro Jahr. In diesem Berichtszeitraum konnten mehr als 11.000 Besucher begrüßt werden. Die jährlich wiederkehrenden Haupttagungen sind

- das Aufbereitungstechnische Seminar,

- der Österreichische Bergbautag sowie

- die Herbstveranstaltung.

\subsection{Fachausschüsse - Exkursionen}

Die Fachausschüsse organisieren jährlich mehrere nationale und internationale Exkursionen innerhalb Österreichs und vor allem nach Deutschland, in die Tschechische Republik, nach Polen, Schweden und Finnland.

Die Exkursionen erfreuen sich besonders in der Studentensektion großer Beliebtheit, bieten sie doch Gelegenheit, die europäischen Partneruniversitäten und ihre Traditionen 
kennenzulernen und internationale Kontakte zu knüpfen. Neben dem Austausch von Wissen - am Programm stehen Besuche von Bergwerken und Museen - ermöglichen Firmenbesichtigungen einen Einblick in die Wirtschaft des Gastgeberlandes.

Die Fachausschüsse pflegen durch Erfahrungs- und Informationsaustausch die Vermittlung von Anregungen und fördern die technisch-wissenschaftliche Entwicklung sowie die schnelle Verarbeitung neuer Erkenntnisse. Fachausschüsse dienen damit gleichzeitig der Ausbildung und Weiterbildung der Verbandsmitglieder.

\subsection{Herausgabe eines eigenen Schrifttums}

Die Berg- und Hüttenmännischen Monatshefte sind das technisch-wissenschaftliche Organ der Montanuniversität Leoben, der ASMET Research GmbH (Austrian Society for Metallurgy and Materials) und des BVÖ (Bergmännischer Verband Österreichs). Sie erscheinen $12 \mathrm{mal}$ pro Jahr in Kooperation mit dem Verlag Springer Wien - New York, derzeit im 165. Jahrgang.

Die Hefte werden abwechselnd von ASMET und BVÖ inhaltlich gestaltet und stehen den Mitgliedern nicht nur in gedruckter Form, sondern auch digital zur Verfügung. Die digitale Nutzung und der Zugriff auf die online publizierten Artikel ist in ständigem Wachstum begriffen, was durch die "Open Access" Publikation zahlreicher Beiträge noch gefördert wird.

Neben der Veröffentlichung von Vorträgen verschiedener Veranstaltungen und einem breiten Spektrum von wissenschaftlichen Artikeln akademischer Autoren besteht auch für junge Autoren die Möglichkeit, über ihre Forschungstätigkeit zu berichten und die Themen ihrer Diplomarbeiten bzw. Dissertationen zu präsentieren.

\section{Nationale und internationale Verbindungen}

Die Sektion Bergbau und Energie ist im Bundesministerium für Landwirtschaft, Regionen und Tourismus angesiedelt:

„Vom Grundsatz der Nachhaltigkeit getragen verfolgt der Bergbau die volkswirtschaftlich beste Bereitstellung von Energieträgern und Rohstoffen unter Bedachtnahme auf Versorgungssicherheit, Kostengünstigkeit und Umweltsowie Sozialverträglichkeit.

Unsere Gesellschaft ist existenziell von einer funktionierenden Rohstoffversorgung abhängig, gepaart mit dem Wunsch nach Integrität von Natur und Umwelt" [3].

WKO (Wirtschaftskammer Österreichs) Traditionell besteht eine enge Zusammenarbeit des BVÖ mit den facheinschlägigen Gruppierungen der WKO, wie z. B. dem

- Fachverband Bergwerke und Stahl,

- Fachverband Stein- und keramische Industrie,

- Forum mineralische Rohstoffe.

\subsection{Internationale Verbindungen}

Die Probleme des Bergbaues lassen sich nicht auf ein Land begrenzen, der internationalen Zusammenarbeit auf diesem Gebiet kommt eine besondere Bedeutung zu.

Der BVÖ pflegt besonders die Beziehungen zu folgenden Partnerorganisationen:

- Gesellschaft der Metallurgen und Bergleute e. V. (GDMB)

- Deutscher Markscheider Verein (DMV e.V.)

- Verband Bergbau, Geologie und Umwelt e. V. (VBGU)

- European Federation of Geologists (EFG)

\section{Wegweisende Entwicklungen für die Zukunft - Kooperation mit EFG}

Im Mai 2019 ist der BVÖ als 27. Mitglied der European Federation of Geologists (EFG) beigetreten. Die EFG ist eine NonGovernmental Organisation mit Sitz in Brüssel und vertritt die Interessen von mittlerweile 47.000 Mitgliedern.

Die Mitgliedschaft beim EFG bedeutet für die Mitglieder des BVÖ, sich an europäischen Forschungsprojekten zu beteiligen, Meinungen zu aktuellen rohstoffrelevanten Entwicklungen abzugeben, die bei der Europäischen Kommission oder dem Europaparlament vorgebracht werden können, sowie auch den Funktionstitel „European Geologist” (EurGeol) zu erlangen, der allen Geo- und Montanwissenschaftern zusteht.

Für „Eurogeologists“ besteht darüber hinaus die Möglichkeit, den Funktionstitel "Competent Person“ zu erlangen. Grundvoraussetzung dafür ist eine bereits erfolgte Anerkennung als „Eurogeologist“. Börsennotierte Unternehmen sind verpflichtet, Expertisen nach dem mittlerweile international üblichen NI-43101 - Standard vorzulegen. Solche werden aber nur anerkannt, wenn sie von einer "Competent Person" verfasst wurden.

Die Mitgliedschaft des BVÖ bei der EFG berechtigt seine Mitglieder auch, an EU-finanzierten Projekten mitzuwirken. Derzeit laufen folgende Projekte:

- VAMOS: Viable and Alternative Mine Operating System

- UNEXMIN: Autonomous Underwater Explorer for Flooded Mines

- CHPM2030: Combined Heat, Power and Metal extraction from ultra-deep ore bodies

- INFACT Innovative, Non-invasive and Fully Acceptable Exploration Technologies

- MinLand

- Intermin

- Era-Min

Bei der EFG sind neun Panels of Experts eingerichtet, deren Aufgabe es ist, als beratendes Gremium für die Europäische Kommission bzw. das Europäische Parlament zu fungieren. Die Fachgremien umfassen die Themenbereiche

- $\mathrm{CO}_{2}$ Geological Storage

- Education

- Geotechnics 
- Geothermal Energy

- Hydrogeology

- Natural Hazards and Climate Change

- Minerals and their sustainable use

- Soil protection

- Oil and Gas

Mitgliedern des Bergmännischen Verbandes ist es nunmehr auch möglich, in diesen Gremien mitzuarbeiten und die Interessen zu vertreten. Über die EFG können somit entsprechende Anträge bzw. Stellungnahmen auch an die Europäische Kommission bzw. das Europäische Parlament herangetragen werden [4].

\section{Ausblick}

In seiner 70jährigen Geschichte spannt sich der Bogen des Bergmännischen Verbandes Österreichs von einer Berufsvertretung der Bergingenieure der Montanistischen Hochschule Leoben über seine Erweiterung für Unternehmen und Bergfachleute österreichweit bis zur Mitarbeit in rohstoffwirtschaftlichen Gremien in ganz Europa.

Das gemeinsame Ziel ist eine gesicherte europäische Rohstoffversorgung und die dazu erforderliche erstklassige Ausbildung junger Ingenieure, wissenschaftlicher Informationsaustausch und technische Zusammenarbeit von Universitäten, Unternehmen und Behörden. Der BVÖ leistet dazu einen wesentlichen Beitrag.

Funding. Open access funding provided by Montanuniversität Leoben.
Open Access Dieser Artikel wird unter der Creative Commons Namensnennung 4.0 International Lizenz veröffentlicht, welche die Nutzung, Vervielfältigung, Bearbeitung, Verbreitung und Wiedergabe in jeglichem Medium und Format erlaubt, sofern Sie den/die ursprünglichen $\mathrm{Au}$ tor(en) und die Quelle ordnungsgemäß nennen, einen Link zur Creative Commons Lizenz beifügen und angeben, ob Änderungen vorgenommen wurden.

Die in diesem Artikel enthaltenen Bilder und sonstiges Drittmaterial unterliegen ebenfalls der genannten Creative Commons Lizenz, sofern sich aus der Abbildungslegende nichts anderes ergibt. Sofern das betreffende Material nicht unter der genannten Creative Commons Lizenz steht und die betreffende Handlung nicht nach gesetzlichen Vorschriften erlaubt ist, ist für die oben aufgeführten Weiterverwendungen des Materials die Einwilligung des jeweiligen Rechteinhabers einzuholen.

Weitere Details zur Lizenz entnehmen Sie bitte der Lizenzinformation auf http://creativecommons.org/licenses/by/4.0/deed.de.

\section{Literatur}

1. Fettweis, G.B.L.: 25 Jahre Bergmännischer Verband Österreichs, BHM Berg- und Hüttenmännische Monatshefte vereinigt mit Montan-Rundschau 120 (1975), H. 4, S. 133

2. Fettweis, G.B.L.: Rückblick auf die Entwicklung des Bergmännischen Verbandes Österreichs in den Jahren 1950 bis 2010, BHM Berg- und Hüttenmännische Monatshefte 155 (2010), S. 456-466

3. https://www.bmlrt.gv.at/bergbau.html (Zugriff 07.02.2020)

4. Weber, L.: BVÖ ist der European Federation of Geologists (EFG) beigetreten: Anerkennung als European Geologist (EurGeol) bzw. Competent Person (CP) ab sofort möglich, BHM Berg- und Hüttenmännische Monatshefte 164 (2019), H. 12, S. 563

Hinweis des Verlags. Der Verlag bleibt in Hinblick auf geografische Zuordnungen und Gebietsbezeichnungen in veröffentlichten Karten und Institutsadressen neutral. 